\title{
Experiments on Pitching Plates: Force and Flowfield Measurements at Low Reynolds Numbers
}

\author{
Kenneth Granlund ${ }^{1}$ and Michael $\mathrm{OL}^{2}$ \\ U.S. Air Force Research Laboratory, Wright-Patterson Air Force Base, OH 45433, U.S.A. \\ Luis Bernal ${ }^{3}$ \\ University of Michigan, Ann Arbor, MI 48109, U.S.A.
}

\begin{abstract}
We discuss implementation of prescribed-motion rigs, dye injection and direct force measurement for high frequency, high-amplitude smoothed linear pitch ramps of flat-plate models in a water tunnel. We extend recent results on 2D (wall to wall) flat plates pitching from $0^{\circ}$ to $90^{\circ}$, to rectangular and elliptical (Zimmerman planform) plates of aspect ratio 2. Surveying a range of pivot point locations and pitch rates, we compare the development of leading edge vortices, tip vortices and trailing edge vortices, with trends in lift coefficient and drag coefficient history. The lift peak is seen to correlate with the maximum size of the leading edge vortex, prior to its shedding and downstream convection. Tip vortices evolve more slowly than leading edge vortices, in the sense that a tip vortex remains coherent and nearly attached to the wingtip until well past leading edge vortex saturation and shedding. Peak lift is delayed to progressively higher angles of attack as the reduced frequency is increased. And the non-circulatory or apparent-mass forces are evidently additive to the circulatory aerodynamic forces, implying applicability of linear superposition.
\end{abstract}

\section{Introduction}

In unsteady aerodynamics one is principally concerned with the relationship between the boundary conditions of moving bodies, the flowfield response, and the resulting integrated aerodynamic force and moment history. Here we consider the application to Micro Air Vehicles (MAVs) and to the related problem of biological fliers. Several nuances arise. First, unlike in nearly all other engineered systems, deformations of structures and body outer-mould-lines are large, and can be comparable to the body length scale. This often precludes small-disturbance analysis or linearization. Even for rigid bodies, motion amplitudes in transient or periodic motions can exceed a body length scale. Experiments must duplicate these motions with suitably flexible models and/or imposed motions, such as from robotic model-positioning rigs. Second, time scales associated with flow features over the body become comparable to convective-times and to flightdynamic times scales. In the more usual aeronautical applications, events such as the emergence of flow separation or vortex shedding are much faster than flight-dynamic scales such as the shortperiod mode in aircraft pitching oscillation, because a convective-time is fast and body moments of inertia are large. But in MAVs there is coupling between the various time scales, closely connecting fluid dynamics to flight dynamics, and again complicating attempts at linearization from the viewpoint of theory, and requiring greater attention to model support systems from the viewpoint of experiments. Third, the small scale of MAVs and their operation near ground-level implies a high-disturbance environment. Whereas the usual task of the experimentalist is to produce a maximally quiet flow in ground-test such as wind tunnels to better simulate realistic flight conditions, in MAV applications it is not yet clear what is the "right" spectrum of input turbulence or slower/larger-scale unsteadiness, and how this unsteadiness results in aerodynamic

\footnotetext{
${ }^{1}$ Post-Doctoral Scholar, Air Vehicles Directorate

${ }^{2}$ Senior Aerospace Engineer, Air Vehicles Directorate, Michael.Ol@wpafb.af.mil

${ }^{3}$ Associate Professor, Department of Aerospace Engineering.
} 
response differing from that of quiescent conditions. That is, the maximally quiet flowfield desirable for study of boundary layer receptivity and transition may not be desirable for MAV applications. Gusts, or the reverse problem - aggressive maneuver - result in large excursions of angles of attack and sideslip, often at high dimensionless rate. This leads to the fourth complication: massive separation and return from separation, on a scale more severe than that encountered in manned aircraft, wind turbines and so forth. From the experimental viewpoint this again leads to challenges in supplying representative displacement, rates, kinematic richness and motion fidelity. The problem is even greater for the currently fashionable topic of flapping-wings, where relative displacements are large and unsteadiness is severe, even if the incoming flow is quiescent and the aircraft is not maneuvering. Fifth, and most obvious, is effects of low Reynolds number, resulting in laminar separations and often turbulent transition in free shear layers, sometimes followed by turbulent reattachment. This issue is of two kinds: for cruising fixed-wing MAVs and larger birds or bird-inspired mechanical flappers, one is interested in high L/D and proper tailoring of lifting surfaces for management of laminar separation bubbles, and maximization of leading-edge suction for good propulsive efficiency in flapping-flight. Here the experimental challenge is smooth imposed motions, precise model geometry, clean inflow conditions and accurate near-surface velocimetry, ideally time-resolved. The complementary problem is "insect-type" or aggressive flapping, or aggressive maneuver regardless of vehicle type, where reduced frequencies and amplitudes are large, leading-edge pressure gradients are large and therefore separation is inevitable regardless of airfoil shape or boundary layer state, and instead of boundary layer physics, one is concerned with bulk vorticity shed into the flow. The key experimental challenges are production of the proper kinematics, realization of fluid-structure interaction and aeroelastic scaling, and measurement of frequency-content at sufficient resolution to resolve fast transients due to acceleration.

\section{Experimental Setups and Methods}

\section{Schemes of Model Installation}

Motion rigs can be loosely grouped into three types: bio-inspired flappers, verticalsuspension and horizontal-suspension.

The first category are abstractions of hover-capable natural flyers. Examples include Robofly (Lentink and Dickinson ${ }^{1}$, Lua et al. ${ }^{2}$, and Singh and Chopra ${ }^{3}$. These are the most natural scheme for sweeping-type of rotation coupled with pitching, but require additional actuation to plunge or to translate fore-aft. Motions are limited to semi-strokes of 180-degrees in sweep, followed by motion cessation or stroke reversal. A single wing or a port-starboard pair can be supported, and load cells are typically placed at the wing root. A special case is a continuous rotation machine, typically limited to the single degree of freedom of rotation, with pitch fixed and the would-be flapping wing treated as a propeller. Applications include steady-state measurements after many rotations (Usherwood and Ellington ${ }^{4}$ ), or focus on start-up transients, initial lift overshoot and relaxation to steady state (Jones and Babinsky ${ }^{5}$ ).

The other two categories are derived from aeronautical applications, principally dynamicstall. In vertical suspension, one wing tip is attached to a rotary actuator that pitches the model, and the rotary actuator is in turn attached to a translational actuator for plunge/heave. The model supports are therefore outside of the test section, minimizing intrusion. The resulting rectilinear precludes an biological-type of sweeping motion, but allows pitch to arbitrary high angles. Examples include Buchmann et al. ${ }^{19}$ and Baik et al. ${ }^{10}$ In horizontal suspension, the motion is again rectilinear, but the model is typically supported from its centerplane through struts intruding into the test section. Examples include Rival et al. ${ }^{6}$, Hart and Ukeiley ${ }^{7}$, and OL et al. ${ }^{12}$. In less common variations, the wing is supported from the wingtips, external to the tunnel test section (Brzozowski 
et al. ${ }^{8}$, Bansmer et al. ${ }^{9}$ ). The external support is the cleanest possible aerodynamic installation, and is most suited for cases where accurate assessment of boundary layer physics is important, such as experiments on cruise-conditions of large birds. But because it has a large cantilevered load, it is difficult to produce accurate motions at high frequency. it is difficult to generalize beyond pitch/plunge. The centerplane arrangement is therefore better suited to aggressive motions, but the model support linkages can be intrusive, and raise questions of blockage and interference in the topology of shed vortices.

All three arrangement can be augmented with a third degree of freedom for fore/aft towing, allowing the tunnel to be operated as a tow tank, or to introduce disturbances notionally identified with "gusts" by towing the model fore/aft impulsively. We note, however, that a true gust has a pressure gradient driving the flow, and this is absent if the relative motion is produced by moving the model. On the other hand, model motion would have noncirculatory forces from the imposed acceleration. Therefore accelerating the model with respect to the flow, and accelerating the flow with respect to the model, are not equivalent.

Another area of concern is wall interference effects in strongly vortical flows with nominally 2D conditions. This is the case of a wall-to-wall wing oscillating at large Strouhal number and/or large excursions of effective angle of attack. An example is the PIV results of Bernal et al. ${ }^{10}$ and OL et al. ${ }^{12}$. The former used a vertically-suspended wing of physical aspect ratio 4 , with the light-sheet plane for particle image velocimetry at the $1 / 2$-span. The latter's horizontal centerplane-mounting arrangement implied an optimal light sheet placement at the 3/4-span, to be equidistant from the rig linkages and the test section wall. Physical aspect ratio of the wing was 3. In both experiments the wing tips extended to some nominal distance from the tunnel test section walls, typically less than $1 \mathrm{~mm}$. Comparison of phase-averaged vorticity contours on the plunge downstroke shows a stronger TEV in Baik et al. than in OL et al. Also, the former's results have fine structures in the near-wake that survive phase-averaging, and which are missing in the latter's. One may speculate that these wake structures result from model vibration due to cantilever mounting in vertical suspension, but this is inconclusive. As for the TEV, Visbal ${ }^{11}$ pointed out in a high-fidelity 3D computational study that included sidewall effects, that the difference between the two aforementioned experiments is traceable to the difference in PIV light sheet proximity to the test section sidewall. In going towards the wall, TEV extent attenuates, so that different spanwise sampling location of nominally the same flow may result in different rendition of the TEV. One finds, therefore, that occasionally a computation provides a sort of reverse-validation of experiment, consistent with both being "high-fidelity". Care is required in interpreting results of off-body diagnostics even in configurations of nominally 2D geometry. These difficulties are of course exacerbated in going to genuinely $3 \mathrm{D}$ configurations.

In the following, we document one instance of a three degree of freedom centerplane arrangement, where three translations are coupled to produce one rotation (pitch) about an arbitrary point of rotation, without making hardware changes. We then discuss issue of experimental technique: production of accurate motion history, force measurement, and dye injection.

\section{The AFRL Water Tunnel and Motion Rig}

Experiments were conducted in the U.S. Air Force Research Lab, Air Vehicles Directorate Water Tunnel. The tunnel has $4: 1$ contraction and $46 \mathrm{~cm}$-wide by $61 \mathrm{~cm}$-high test section. Freestream speed range is $3-45 \mathrm{~cm} / \mathrm{s}$, and turbulence intensity is $\sim 0.4 \%$ at $15 \mathrm{~cm} / \mathrm{s}$.

Model motion is effected by a three degree of freedom rig enabling independent control of pitch or rotation, plunge or heave, and "surge" or streamwise-aligned translation. Pitch and plunge are made possible by a pair of electric linear motors mounted vertically on a plate above the tunnel test section. Each motor actuates a vertical "plunge rod", which connects via a bushing to a coupler piece holding the model. The desired angle of incidence and vertical position time history of the model are converted to position commands for each linear motor. The pitch pivot point can be 
varied by suitable choice of phase and amplitude difference in trajectory of front or rear plunge rod. A photograph of the tunnel is shown in Figure 1. More details on the installation are given by OL et $\mathrm{al}^{12}$.

Whenever the pitch pivot point is not coincident with the bushed end of the front plunge rod, there will be a parasitic streamwise displacement of the model, which would be unavoidable unless the front plunge rod were to be allowed to pivot similarly to the downstream one. This is removed using the third degree of freedom, surge, which is achieved using a larger electric linear motor mounted horizontally aft of the pitch-plunge carriage, with 48" peak-to-peak stroke and nominal speed up to $1 \mathrm{~m} / \mathrm{s}$.

The rig's motors are driven by AMC DigiFlex servodrives controlled by a Galil DMC 4040 4-channel card, with user-selected proportional/integral/derivative (PID) constants for each motion channel. PIDs are selected through educated parameter exploration, or more forthrightly, though brute trial and error. Generally PIDs suitable for good motion fidelity for high-acceleration motions result in noise for smoother, lower-acceleration motions, and as with all tuning, the final choice is a compromise. This is made somewhat easier by selecting linear motors and plunge-rods whose own mass is much higher than the mass of test article or of the expected aerodynamic loads. In so doing, the PIDs are essentially tuned for to rig to move itself, and become largely independent of the load.

\section{Motion Definition: the Linear Pitch Ramp}

The pitch motion studied here is nominally constant-rate, or zero acceleration. But for this to be literally true, pitch acceleration would be a delta function at $\alpha=0^{\circ}$, pitch and deceleration would be a delta function at $\alpha=90^{\circ}$. Instead, smoothing transients are applied. A sine wave of 5\% amplitude $\left(\alpha=4.5^{\circ}\right)$ is tangent to the pitch onset, and an analogous wave tangent to the pitch cessation, is first applied. This smoothes the motion, but not its higher derivatives. To obtain $\mathrm{C}^{\infty}$ smoothing, a hyperbolic-cosine function suggested by Eldredge et al. ${ }^{13}$, similar in shape to the aforementioned sinusoids, is fitted to the pitch ramp motion. This is shown above and below the dashed lines in Figure 1. Parameter studies on smoothing transients for pitch-ramp motions have been considered before; see for example Koochesfahani and Smiljanovski ${ }^{14}$. Smoothing transients not only make a given nominally constant-rate motion realizable in an experiment, but are also important from the viewpoint of noncirculatory or acceleration-dependent aerodynamic forces; this is the critical distinction between the present measurements and the traditional view on the role of startup transients. Noncirculatory forces are due to accelerating the ambient fluid around the moving body, and are not related (at least, not directly) to vorticity production. Noncirculatory forces are zero during the constant-rate portion of the pitching motion, but can be large during the smoothing transients, as they are proportional to the square of the effective motion frequency. In principle, different smoothing transients could be applied to the same motion, and different noncirculatory force contributions will result. We also note that since Reynolds number is kept constant $(\sim 10,000)$ while reduced-frequency of pitch is varied, the physical motion takes more convective times when the reduced frequency is slower. The upper limit for maximum attainable reduced frequency, for a given Reynolds number, is excitation of the model/sting/balance natural frequency, which confounds measurement of noncirculatory aerodynamic forces with oscillations in the apparent aerodynamic force coefficients due to structural vibrations. 

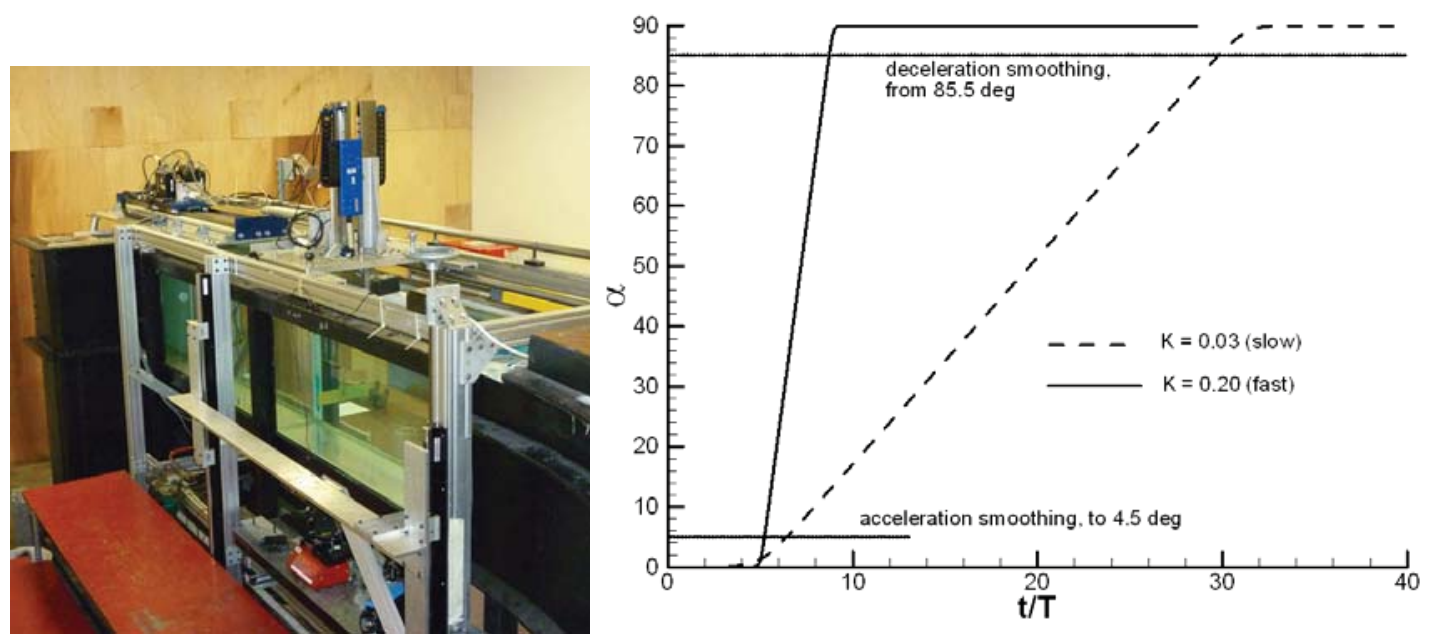

Figure 1. Photograph of water tunnel test section, showing 3-linear-motor motion-rig mounted above test section (left); schematic of pitching motion: angle of attack vs. convective time, for typical "fast" and "slow" pitch rates(right).

The motions are constructed with an algorithm that converts desired pitch/plunge/surge history, subject to smoothing transients and various safeties such as motion range limiters, into a sequence of velocity increment commands that the Galil controller sends at every step of its internal clock. This is therefore not a real-time or dynamic-feedback system, with feedback limited to servo action from the motors' optical encoder tapes. The controller can be programmed to send a TTL trigger pulse, useful for synchronizing the force balance to the motion history, or for firing a PIV laser. The triggers is keyed off relative motion in any of the channels, and for reliable triggering, time $\mathrm{t}=0$ begins from a small motion preceding the actual motion of interest; typically this is a $1 \%$ of chord streamwise surge.

\section{Test Articles}

One motivation for the present work is attempt at fundamental understanding of how motion rates affect vorticity production and departure of lift and drag coefficient from the quasisteady case. A more practical application is to the maneuver of "perching" 15 , which combines rapid pitch-up and free-stream deceleration. However, recent work on "perching" motions of 2D flat plates ${ }^{16}$ has shown minimal influence of streamwise deceleration on lift and drag coefficient history, at least until the angle of attack where coefficient peak values are achieved. This suggests that while careful study of perching maneuvers does require representation of free-stream deceleration, especially for calculations of stability derivatives and other matters of flight control in deep-stall, the process of vortex formation and shedding can be adequately represented even with a constant free-stream. We rely on this rationale to extend the work on pure-pitch of a 2D flat plate, to plates of low aspect ratio $(A R=2)$ : a rectangular plate and a Zimmerman (Laitone ${ }^{17}$, Mueller ${ }^{18}$ ) plate. The Zimmerman plate has planform of ellipses describing the leading edge and the trailing edge, joining along their major-axis on the plate $3 / 4$-chord. All plates have round (semicircle) edges. Since one would expect that the scaling of leading edge vortices would vary with the local wing chord, vortex evolution along the span should differ between the two plates, for otherwise identical motions. In all cases, the mean aerodynamic chord was used to determine the reduced frequency of pitch, $K=c \dot{\theta} / 2 U_{\infty}$. 

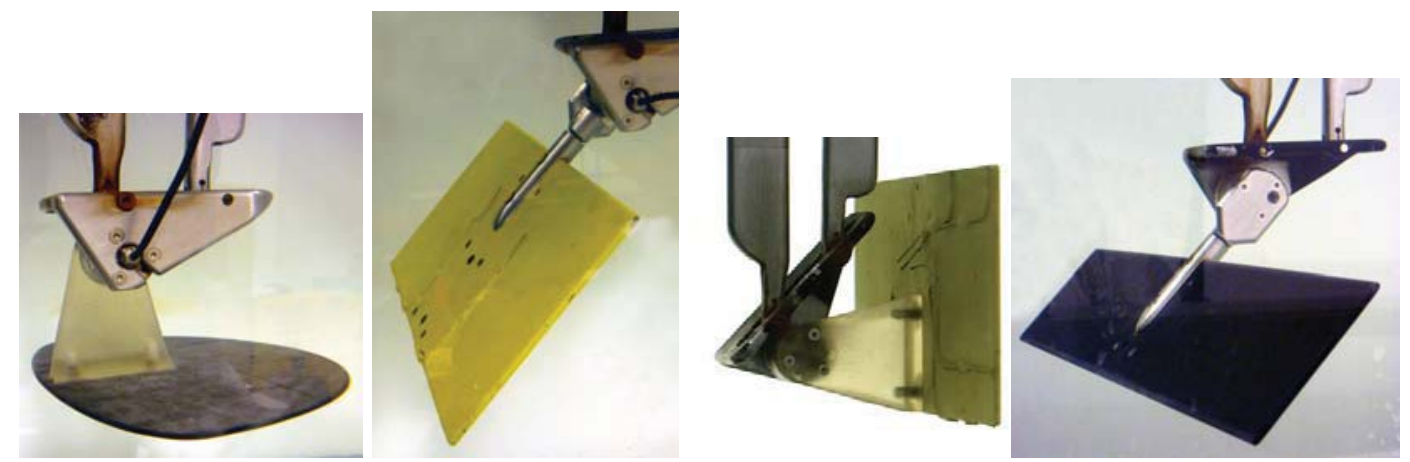

Figure 2. Model installations in water tunnel: $A R=2$ Zimmerman plate with strut mount (left); $A R=2$ rectangular plate with sting mount (middle left) and with strut mount (middle right), and wall-to-wall rectangular plate with sting mount (right). The Zimmerman plate's installation is here with zero angle of attack offset, while the other three have 45-degree angle of attack offset. Flow is from left to right, and tunnel test section walls are not visible.

\section{Force Measurement and Data Post-Processing}

Force data are recorded from an ATI Nano-25 IP68 6-component integral loadcell, oriented with its cylindrical axis normal to the pitch-plunge-surge plane. The loadcell is visible in the leftmost portion of Figure 2, joining the triangular steel plunge rod coupler and the plastic strut to the flat plate model. Load cell strain gage electrical signals are A/D converted in an ATI "NetBox" interface and recorded over Ethernet LAN UDP protocol to a computer using a Java application. The time-base of the ATI NetBox is slightly inaccurate with the clock operating at a factor of 1.0023 too fast with respect to physical time. This is corrected for in post-processing of data. A disadvantage of the IP68 waterproofing of the loadcell is that it is sensitive to immersion depth in the cylindrical axis direction. Because this direction is normal to the plane of the motion of symmetrical models, the hydrostatic force will not affect either normal-, axial force or pitching moment. Force and motion data are synchronized by polling for the trigger signal every $10 \mathrm{~ms}$ and starting the data recording when initial trigger is detected.

All post-processing is done in Matlab. Before each run, the loadcell is zero-biased at model $\theta=0^{\circ}$, which is adjusted to horizontal with a bubble level. A static tare sweep over $90^{\circ}$, either $45^{\circ}<\theta<45^{\circ}$ or $0^{\circ}<\theta<90^{\circ}$ depending on the subsequent dynamic motions, is performed with 500 samples of data every $2^{\circ}$. Linear fits of tare forces in model axial- and normal directions, as well as tare pitching moment to recorder data are shown in Figure 3. Because the pitch angle is known throughout the motion, and the position error is negligible, the static axial force, normal force and pitching moment due to static model/sting/mount weight can be subtracted from the unsteady force data. The dynamic inertial force and moment are only dependent on motion acceleration. However, so are the non-circulatory fluid loads. These can be approximated by a cylinder of fluid encompassing the model undergoing the same motion acceleration. For the current $A R=2$ flat plates, the model mass of $0.15 \mathrm{~kg}$ is $6 \%$ of the fluid "equivalent added mass" of $2.5 \mathrm{~kg}$. Since the model acceleration cannot be determined with $6 \%$ accuracy, any attempt to remove the model mass inertial force in a dynamic maneuver is likely to introduce just as large inertial force errors. This step is thus ignored in the post-processing. 

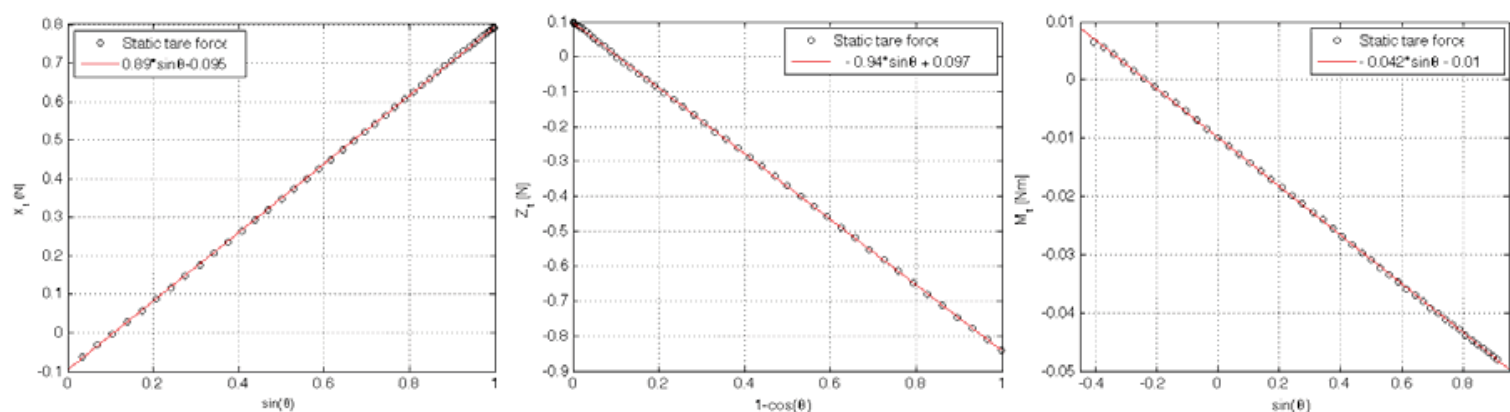

Figure 3. ATI Nano-25 load cell post-processing: Linear fit to tare axial- (left), normal (middle) force and pitching moment (right)

The force and moment signals are then filtered in three steps. This first Is a low-pass filter in the ATI NetBox at $\mathrm{f}=73 \mathrm{~Hz}$, to avoid introducing noise not correlated with motion force data, but without attenuating important fast non-circulatory "load spikes". These are on the order of a fifth as slow as the $-3 \mathrm{~dB}$ (half amplitude attenuation) point of the filter for the fastest pitch ramp corner acceleration. The second step uses a moving-average of 11 points to smooth the data while preserving as much of the non-circulatory load spikes as possible. This smoothing also makes the $\mathrm{s}$ more numerically stable the final step, which is a $6^{\text {th }}$ order Chebychev II $15 \mathrm{~Hz}$ low-pass filter with $20 \mathrm{~dB}$ attenuation of the stopband. It is chosen for maximum passband flatness and high rejection of structural eigenfrequencies which may be just above the desired force frequency information range. To preclude time-shift of useful data in the passband, the forward-backward filtering technique with the Matlab 'filtfilt' command is used. Finally, the forces in the model coordinate system are transformed to the tunnel freestream coordinate system of lift and drag and normalized by freestream dynamic pressure and planform area. Time is normalized by freestream speed and mean aerodynamic chord into convective time.

\section{Comparison of blockage from different model mounts}

The desire to maximize the system structural natural frequency implies a large and stiff model support arrangement, in conflict with the competing desire to minimize blockage and disturbance of the flowfield due to the model support. This is of course a ubiquitous problem in wind tunnel/water tunnel testing, but in the present context the question is how blockage varies across a large range of angle of attack, over which the model itself sweeps a large variation of projected area. Large, intrusive support systems may produce interference unacceptable for low angle of attack drag polar measurements, but may be entirely adequate for assessing broad trends in going from $\alpha=0^{\circ}$ to $\alpha=90^{\circ}$. Figure 4 shows low angle of attack static lift and drag trends for the $\mathrm{AR}=2$ rectangular plate at $\mathrm{Re}=20 \mathrm{~K}$, for the sting mount with zero pitch offset, sting mount with 45 degrees pitch offset, and the same two conditions for the strut mount. The strut mount with 45degree offset is seen to have the largest mount-interference, in the sense of a positive offset in lift and drag coefficients. Theses deltas relative to the other runs - approximately 0.07 and 0.025 - are unacceptably large for studies of static aerodynamics at low angle of attack. Even more obviously, a flat plate at geometrically zero incidence should not be producing a lift coefficient of 0.14 . But as will be shown below, such deltas are small relative to lift and drag coefficient magnitudes for aggressive maneuvers.

The lift and drag deltas also appear in the dynamic cases. Figure 5 continues Figure 4's comparison of lift and drag for the four mounting arrangements, this time for the nominally $2 \mathrm{D}$ or wall-to-wall plate, for pitch of $\alpha=0^{\circ}$ to $\alpha=45^{\circ}$ (pitch all the way to $\alpha=90^{\circ}$ is only possible with the $45^{\circ}$ - offset mount, and therefore this discussion is limited to $0^{\circ}-45^{\circ}$ motions). For $\mathrm{K}=0.03$, the $45^{\circ}$-offset strut mount again has positive lift and drag coefficient deltas, of amplitudes comparable to those in Figure 4. This offset continues until the lift peak (at $\alpha \sim 22^{\circ}$ ) and drag peak (at $\alpha \sim 36^{\circ}$ ) 
However, for $\mathrm{K}=0.20$, the static deltas are not carried over, and there does not appear to be a simple trend of aerodynamic force coefficient increment vs. mounting arrangement, valid for all reduced frequencies.
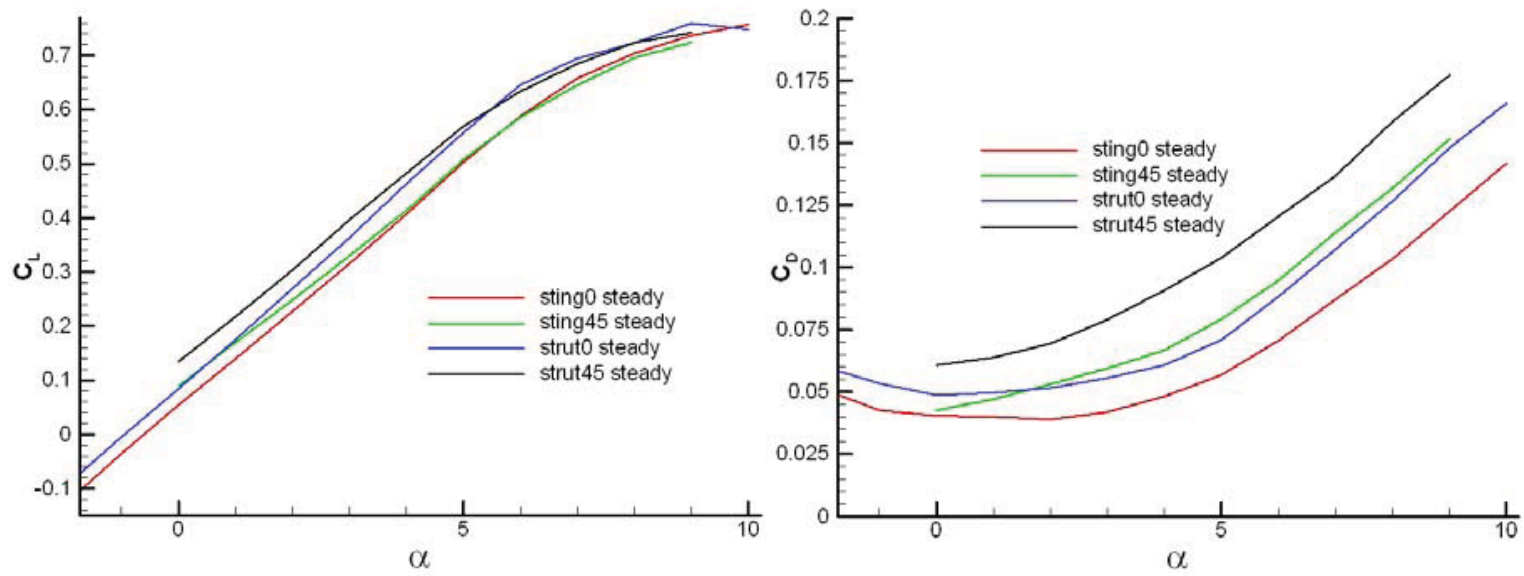

Figure 4. Static sweeps, lift (left) and drag (right), $R e=20 \mathrm{~K}, \mathrm{AR}=2$ rectangular plate, with four mounting arrangements: strut and aft-sting, 0 -degree and 45-degree pitch offsets.
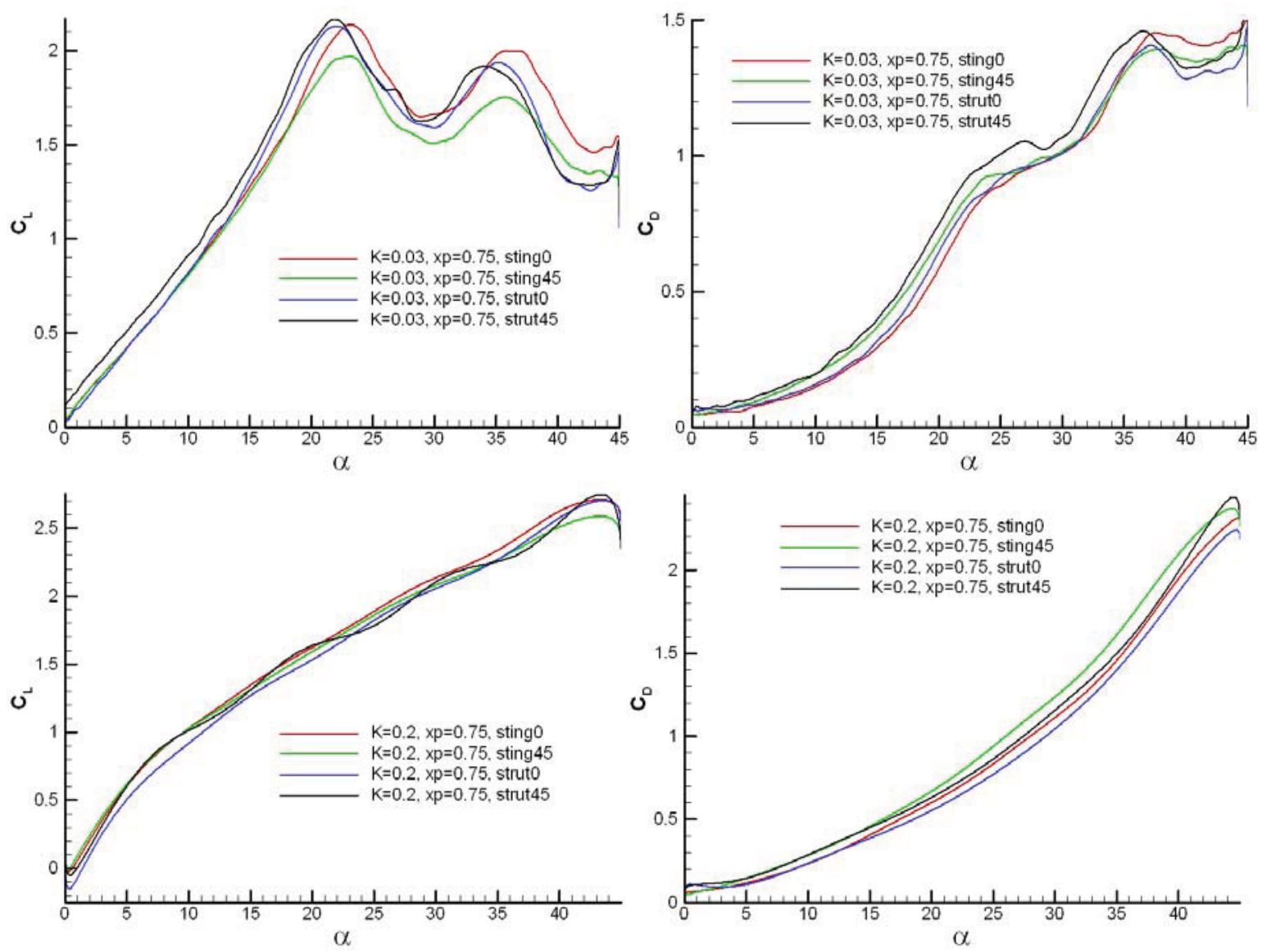

Figure 5. Lift coefficient (left) and drag coefficient (right), $K=0.03$ (top) and $K=0.20$ (bottom), 2D flat plate pitching from $\alpha=0^{\circ}$ to $\alpha=45^{\circ}$, pivoting at $x / c=0.75$, with four different mounting arrangements: aft sting, aft sting with 45-degree offset, vertical strut, and vertical strut with 45-degree offset. 
Discussion of accuracy of force measurement would be incomplete without mention of drift or biases appearing in force coefficient history. Some of these are random, and others are systematic. Biases occur for example when the time-averaged zero-reading after a run with aggressive motions differs from a comparable reading before the motion, as if the experience of the motion "biased" the force balance. For the ATI Nano-25, we systematically find a bias of $0.005 \mathrm{~N}$ in normal and axial force. This is well within the manufacturer's quoted tolerance of $1 \%$ of fullscale reading. But one should note that at $\mathrm{Re}=10 \mathrm{~K}$, for the $\mathrm{AR}=2$ model, $0.005 \mathrm{~N}$ corresponds to an aerodynamic force coefficient of 0.05 . Assuming a static lift curve slope of $\pi$, this is comparable to a static angle of attack uncertainty of $1^{\circ}$ - which, again, is unacceptably large for tests of static aerodynamic performance, but quite acceptable for aggressive motions. Of course, as Reynolds number doubles, the coefficient-equivalent drift attenuates by a factor of 4 . So in Figure 4, which is at $\mathrm{Re}=20 \mathrm{~K}$, the ratio of balance drift amplitude to measured delta due to mount interference, is $<0.1$.

\section{Motion Fidelity}

Unlike minor offsets, biases or uncertainties in the force measurement, good accuracy in imposed model kinematics is essential for all reduced frequencies, amplitudes and Reynolds numbers. Generally one plots aerodynamic force coefficient vs. the theoretically prescribed angle of attack history, as the actual angle of attack history would suffer from high-frequency undulations from vibration, elastic deflection of linkages and so forth. Measurement of actual model motion would require a photogrammetry technique, laser vibrometry and so forth - and is not attempted here. We can, however, compare the commanded vs. attained model position history from interrogating the optical encoder tapes of the three linear motors, operating on the assumption that the main source of positioning error would be motor jitter, and not structural deflection of linkage members.
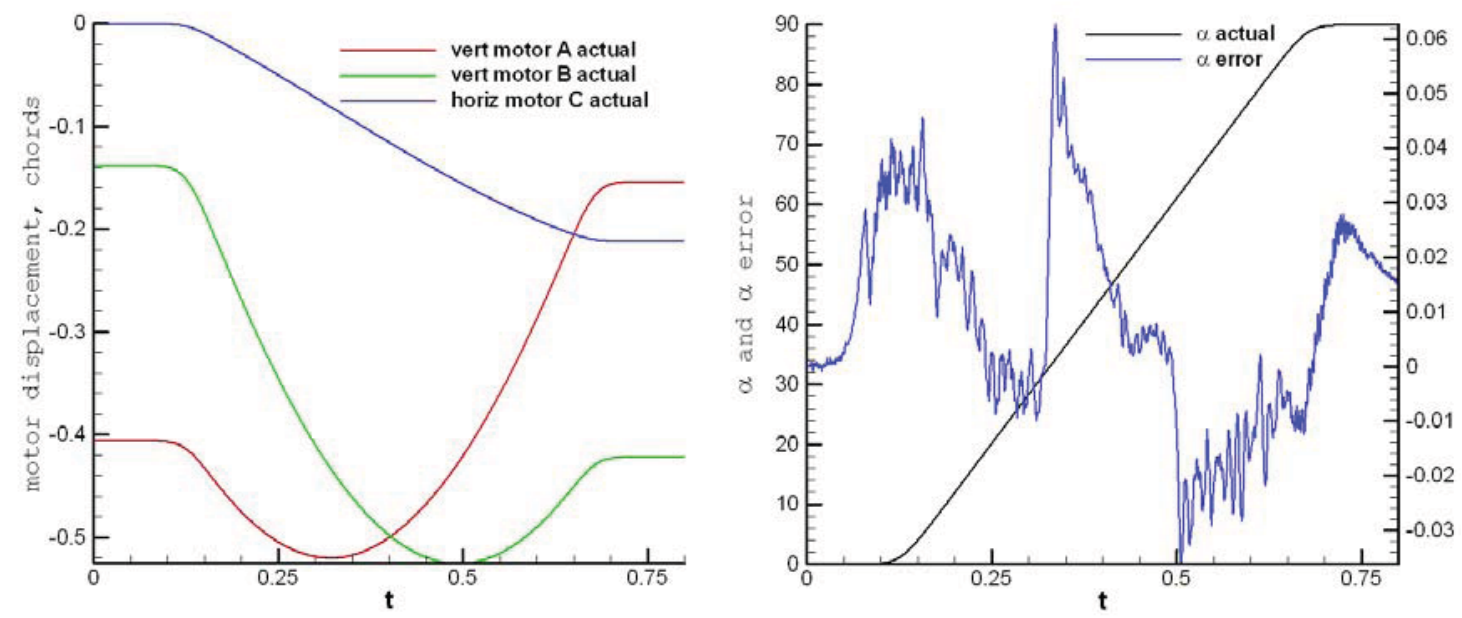

Figure 6. Rig and model kinematics: actual trajectory of the three linear motors vs. convective time, in fractions of $A R=2$ rectangular plate chord (left); actual angle of attack history and error in angle of attack (right). Motion is pivoting about the leading edge, strut mount, $A R=2$ rectangular plate, $R=$ $10 K . K=1.0$, and therefore the entire motion occupies $\sim 0.5$ convective times.

Because the rig's principle of operations is conversion of three translating motions - in the case of a pure pitch - to one rotational motion, how errors in the three motors propagate into an error in angle of attack depends on the spacing between the tips of the two vertical motors' plunge rods, which is $44.32 \mathrm{~mm}$. To achieve a 90-degree rotation of the model about a point on its chord line, the three motors perform translations on the order of 0.5 chords of the $\mathrm{AR}=2$ model (chord $=$ $117 \mathrm{~mm}$ ). Figure 6 plots the position history of the three motors, angle of attack history and angle 
of attack error history. Peak errors in angle of attack are less than 0.1 degrees, at the linear motor carriages. We consider these to be sufficiently low as to disregard the distinction between theoretical (commanded) angle of attack, and actual achieved angle of attack. But to reiterate, this assertion is predicated on assumption of a perfectly rigid model and strut.

\section{Load Cell Frequency Response}

For massively unsteady problems, one often finds lift coefficients on the order of 5, 10 or higher - which means that even at small dynamic pressure and with physically small models, relatively large force balance load capacity is needed. But this can imply inadequately low resolution for more conventional tests such a static sweeps at low angle of attack. Thus the same balance can not be used for aerodynamic performance-type of testing (drag polars, pitch static stability) and unsteady-maneuver testing (rapid pitch-up, response to sharp gusts) if good accuracy is desired in both.

Water of course allows for much higher dimensionless frequency tests than in air typically, by a ratio of 17 , which is the approximate kinematic viscosity ratio between air and water at room temperature. Alternatively, the same dynamic pressure is achieved in water as in air, if the former's free-stream speed is some $30 \mathrm{X}$ less - very convenient for either slowing down the experiment or improving force magnitudes for better force measurement accuracy. However, one has to consider the frequency response of the model-sting-support-balance system. Balance stiffness increases with load capacity and decreases with resolution; typically it is on the order of $\mathrm{KHz}$ and can be ignored. However, the overall system stiffness, which is an upper-bound for frequency resolution in the aerodynamic measurement, is greatly affected by the apparent-mass of water. This is in direct relation to the noncirculatory load on an accelerating model. Even if the nominal motion is unaccelerated, there will be vibrations and startup transients.

Figure 7 compares the results of "strike tests", where the model - in this case the AR=2 rectangular plate - is literally struck by a blunt object. The alternative is to command a smallamplitude violent maneuver with the motion rig, such as a step in plunge. But the disadvantage of this approach is that effectively one studies the transfer function of the combination of the rig and the balance/mount/model, instead of merely the latter. Four cases are considered: the aft sting mount and strut mount, both at zero degree offset, with the tunnel filled with water and the rig's motors turned off; the strut case, with the motors energized and holding the model steady; and finally, with the strut, the motors energized and the water drained. Figure 7 plots the response history of the normal-force channel, and the FFT of the response after the initial pulse is removed. Clearly the sting-mount has more compliance than the strut, though the sting is stainless steel while the strut is plastic. The reason is that the strut is a short, direct mount engaging the model near its center of mass, while the sting is a long cantilever. The difference is a first natural frequency of about $24 \mathrm{~Hz}$ for the sting, and about $55 \mathrm{~Hz}$ for the strut. Turning on the rig's motors produces a curious result: the strong, narrow response peak is broadened and shifted to a slightly lower frequency, while a second, smaller peak appears at a frequency of around $18 \mathrm{~Hz}$. One may speculate that the motors produce a sort of damping, absorbing low-frequency inputs, but ring at a frequency of their own. Most striking, however, is the effect of draining the water. The system natural frequency should behave as $f \sim \sqrt{k / M}$, where " $\mathrm{k}$ " is the stiffness (not to be confused with reduced frequency of motion!) and " $\mathrm{M}$ " is the system mass. "M" includes the model, the support system and the metric side of the balance, when the water is drained. But it includes the mass of a circular cylinder of water of the same diameter as the model chord, and same height as the model span, when the tunnel is filled. $M$ in water, vs. $M$ in air, is greater roughly by a factor of 16 - and this has profound implications for the range of frequencies measurable in water tunnel tests, and even for the procedure of doing dynamic tares. In fact there is no longer a discernable structural frequency peak in the water-drained case. Instead, there is a broadband response, consistent with the assertion that a structural peak would move to much higher frequencies. The 
absence of water, on the other hand, may have the effect of attenuating damping of vibration produced by the strike.
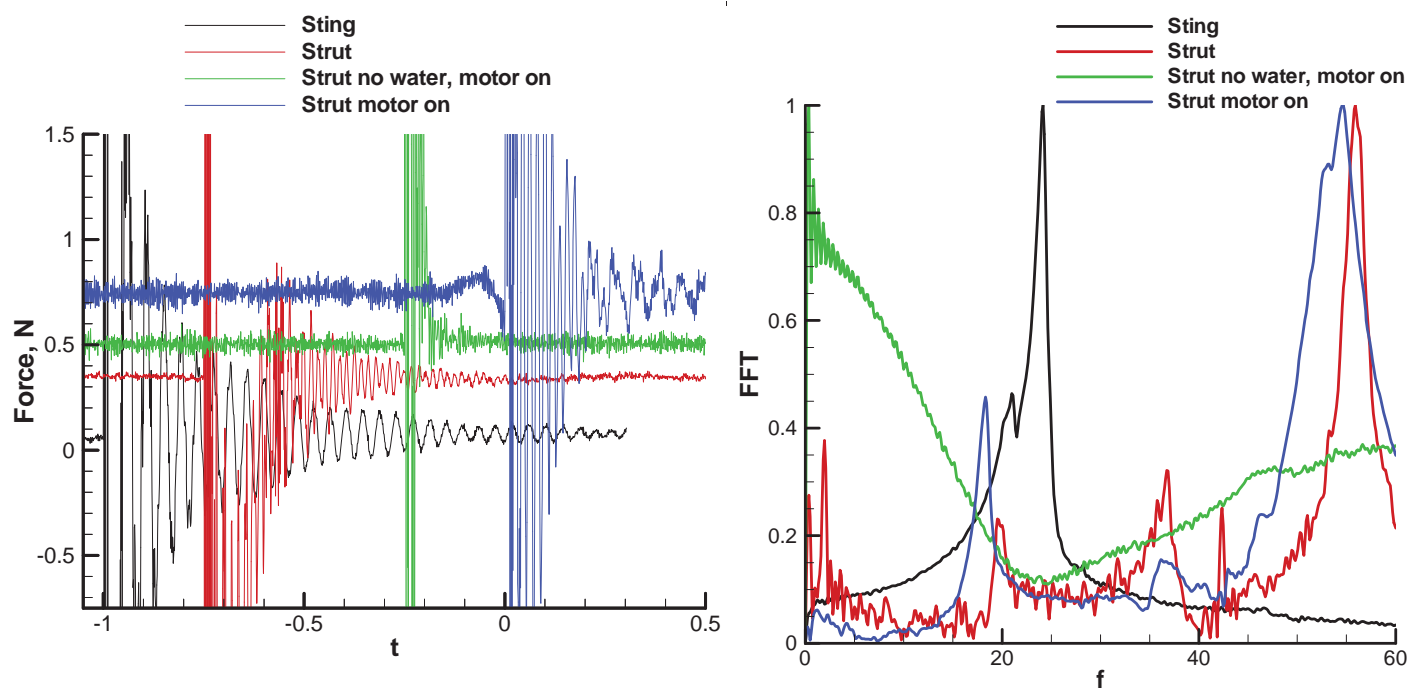

Figure 7. Time-trace of normal force (left; four cases mutually offset by $0.25 \mathrm{~s}$ time difference and $0.25 \mathrm{~N}$ force difference for clarity) and frequency content (right) of strike tests for $\mathrm{AR}=\mathbf{2}$ plate.

\section{Dye Injection}

The typical objective of flow visualization by dye injection in low-Reynolds number unsteady aerodynamics is a to achieve a qualitative surrogate for vorticity, placing faith in the assertion that vortex-cores are low pressure regions which would entrain higher concentrations of dye. Then dye injection would be used for large parameter studies for subsequent downscoping of a few salient cases to forays with more sophisticated methods, such as Particle Image Velocimetry and its various 3D extensions. An example of the ordering of going from dye injection to sophisticated PIV measurements is the tomographic PIV experiment of Buchmann et al. ${ }^{19}$, resolving the vortical structures of the near-wake behind a pitching 2D plate outlined in the dye injection results of $\mathrm{Ol}$ et al. ${ }^{23}$

For nominally 2D problems with nonzero free-stream, such as wall-to-wall plates or airfoils with strong TE and LE vortices, injection of dye at the LE or TE can be an excellent proxy for outof-plane vorticity component (that is, spanwise vorticity); see for example the dye injection and 2D PIV comparison on OL et $\mathrm{al}^{12}$. For zero free-stream, modeling for example a hovering flapping wing, there is no preferred direction into which to entrain dye before motion onset, but during the motion the strain and diffusion can be very high, whence profuse dye flow rates may be required to obtained images with good contrast. Dye injection is still successful for start-up problems, such as a plate commencing a pitching or translating motion. But as the motion evolves, diffusion becomes more problematic. This is evident or example in Figure 8 and Figure 9 for the pitching rectangular and Zimmerman plates, respectively. As the plate approaches the conclusion of its motion, $\alpha \sim 90^{\circ}$, dye streaklines no longer follow discernable flowfield features such as vortex cores.

In the present experiments, dye injection was by a mixture of food coloring dye, milk and methyl alcohol, to achieve density-match with the ambient water. Density matching is important for flow speeds below nominally $10 \mathrm{~cm} / \mathrm{s}$. Since test articles are typically mounted with the suction-side towards the tunnel test section bottom, a dye mix heavier than the ambient water in an otherwise attached flow will appear as suction-side boundary layer thickening. Inversely, dye mix lighter than water may make a slightly separated flow appear to be attached, as the dye streak will cleave closer to the model's suction side. 
How intrusive is dye injection? Since the dye at the injection point is essentially a jet in crossflow, one asks whether this jet promotes early transition or causes other spurious effects. A qualitative argument for relatively nonintrusive dye injection is when the dynamic pressure of the dye stream is small compared to the pressure difference between free-stream and viscous cores of the vortices that one wishes to visualize. But this is hard to quantify in practice. Instead, one tries different dye volumetric flow rates and seeks an optimum of maximum contrast and minimum variation of resulting flowfield across a range of injection rates. This of course will vary from leading-edge, wingtip and trailing-edge injection points. Dye infusion rate is adjusted to limit the flow rate from every tube to $<30 \%$ of nominal free-stream speed.

In the present implementation, a positive-displacement pump with a prescribed volumetric infusion rate introduced the dye through a set of $0.5 \mathrm{~mm}$ internal-diameter rigid lines glued to the surface of the plate. The positive-displacement method allows for more control than the principal alternative - gravity-feed; for comparison between the two, see Yilmaz et $\mathrm{al}^{20}$. For visualizing leading edge vortices, the dye lines run along the plate leading edge, firing outboard. For visualizing the wake and trailing edge vortices, the dye lines exit in the streamwise direction, terminating just upstream of the trailing edge. And for visualizing tip vortices, dye lines run parallel to the wingtip, firing downstream. Dye is injected at the midspan, 3/4 span, 7/8 span, and near the tip, all on the starboard panel of the wing. Tubes intended for leading edge visualization fire in the spanwise outboard direction, while those intended for tip or trailing edge visualization fire in the downstream direction. At low angles of attack, the physical presence of the dye lines disturbs the flow, as they are wall-conformal obstacles.

\section{Results}

\section{Flow visualization: 2D vs. 3D}

Figure 8 and Figure 9 compare the AR=2 rectangular and Zimmerman wings, respectively, for a linear pitch ramp maneuver, $\alpha=0^{\circ}$ to $90^{\circ}$, at reduced frequency $K=0.20$. Side views, showing LEV and TEV sectional cuts, are compared with planform views, which show spanwise development of the LEV, TEV and tip vortex. Pivot points at $\mathrm{x} / \mathrm{c}=0,0.5$ and 1.0 are considered for the rectangular plate, and $\mathrm{x} / \mathrm{c}=0$ and 1.0 for the Zimmerman plate. At this high frequency, development of the leading edge vortex, which is so characteristic of pitch or plunge motions, lags the motion kinematics. In comparing with Granlund et al. ${ }^{16}$, who discuss results for a $2 \mathrm{D}$ plate, the LEV for all $A R=2$ cases is seen to be less coherent, in the sense of greater dye diffusion and less dye entrainment into a definitive vortex core. In comparing LE, midchord and TE pivot points, one finds that LEV development is delayed as the pivot point is taken further aft. Indeed, for the TE pivot point, an LEV first forms on the plate pressure (receding) side, before switching to the suction (advancing) side. But in all cases there is a spanwise flow during the upstroke from inboard towards the centerplane, as identified in Yilmaz et al. ${ }^{20}$ for the related motion of pitch ramp-holdreturn. For the Zimmerman plate, this spanwise flow is weaker than for the rectangular plate, possibly because for the former the spanwise variation of chord results in its own spanwise pressure gradient effect.

The tip vortex is seen to deflect with increasing angle of attack at a slower rate than LEV evolution, in the sense that tip vortex trajectory remains planar relative to the wing chord-plane to angles of attack by which the LEV has already formed, and indeed detached. This suggests, at least notionally, that tip vortex formation-time is longer than LEV formation time, with implications for attenuation of aspect ratio effects for increasing reduced frequency. 

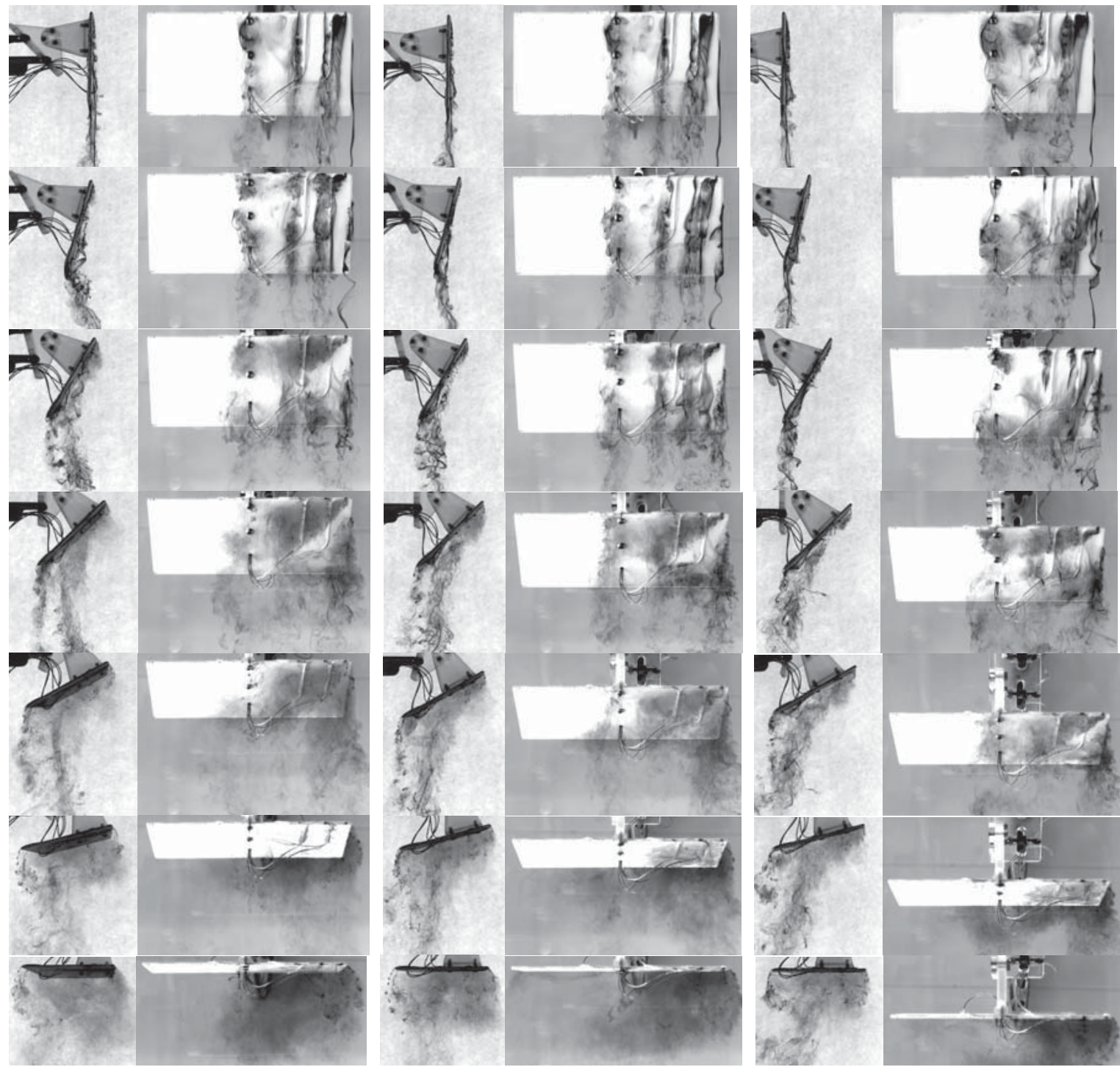

Figure 8. Side view and planform view of rectangular $A R=2$ plate pitching at $K=0.20$, with pivot at leading edge (left), midchord (middle) and trailing edge (right). Snapshots at incidence angles of $0^{\circ}$, $15^{\circ}, 3^{\circ}, \mathbf{4 5}^{\circ}, \mathbf{6 0}^{\circ}, \mathbf{7 5}^{\circ}$ and $90^{\circ}$. Flow in each snapshot is from top to bottom.
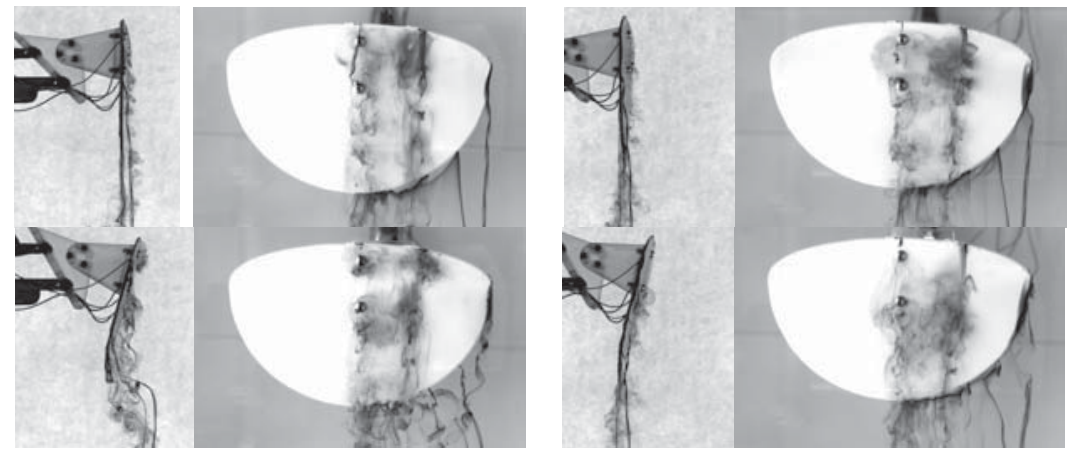

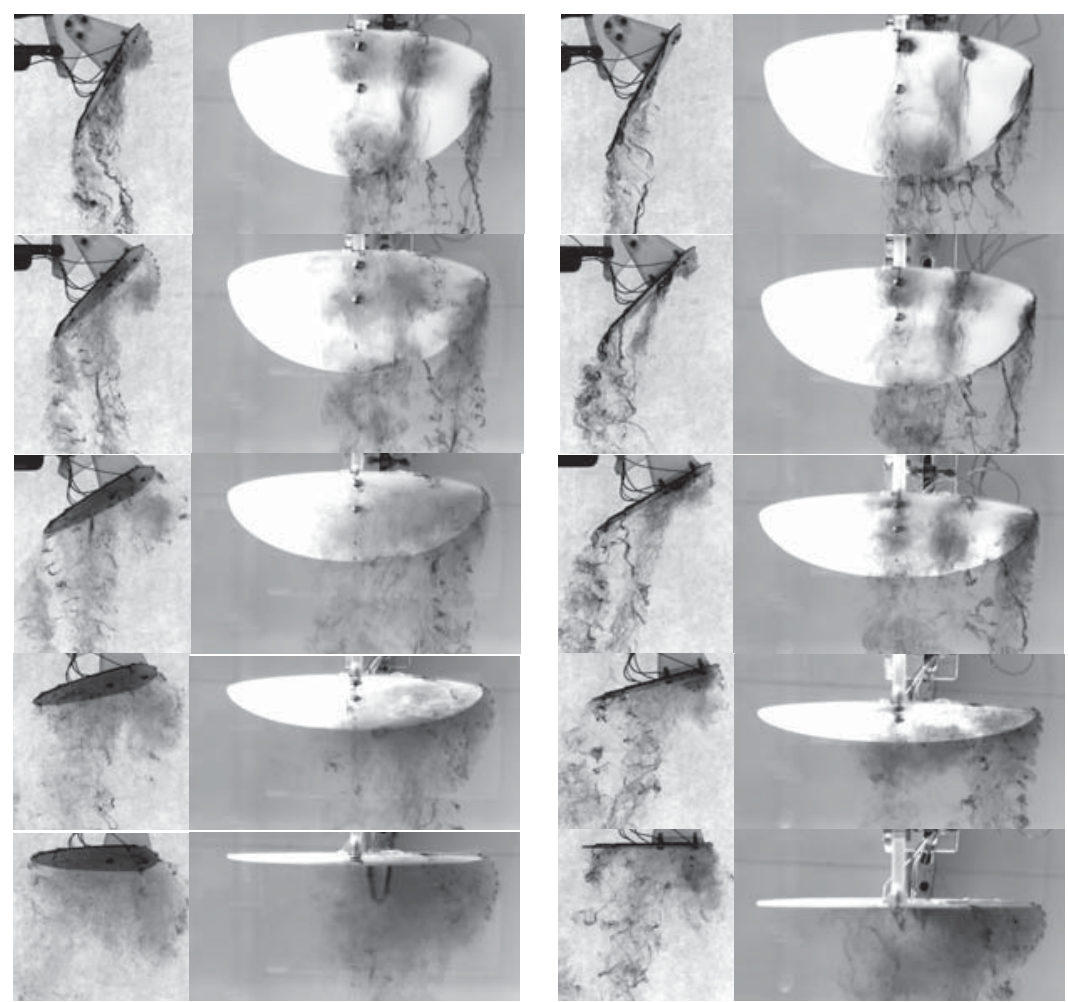

Figure 9. Side view and planform view of Zimmerman $A R=2$ plate pitching at $K=0.20$, with pivot at leading edge (left) and trailing edge (right). Snapshots at incidence angles of $0^{\circ}, 15^{\circ}, 30^{\circ}, 45^{\circ}, 6^{\circ}, 7^{\circ}$ and $90^{\circ}$.

Dye injection is seen to be of decreasing utility as the angle of attack increases, as the diffusive nature of the flow decreases the dye's ability to qualitatively track likely concentrations of vorticity.

\section{Force measurement}

We consider a range of reduced frequencies and pivot point locations, extending the results of Granlund et al. ${ }^{16}$ to reduced frequencies of 1.0, to compare with results of Baik et el. ${ }^{21}$, who considered a cantilevered 2D rectangular plate pitching at $\mathrm{K}=0.2,0.5$ and 1.0. A survey of lift and drag coefficient for various reduced frequencies is shown in Figure 10 for the $A R=2$ rectangular plate. Figure 11 compares the Zimmerman and rectangular plates for a selection of reduced frequencies. Qualitatively, the $A R=2$ plates follow the trends of the $2 \mathrm{D}$ plate, in the sense of (1) a noncirculatory lift increment due to acceleration in startup transient and a similar drag increment due to deceleration in the stopping transient, with the noncirculatory contribution proportional to the square of the reduced frequency; (2) essentially linear increase in lift coefficient with increase in reduced frequency, up to the point of peak lift; (3) delay of peak lift to progressively higher angle of attack with increasing reduced frequency; (4) close following of the lift coefficient with the theoretical lift curve slope ( $=\pi$, for $\mathrm{AR}=2$ wings; cyan dashed curve in Figure 10 and Figure 11) at the smaller reduced frequencies at low angle of attack, but progressive reduction in lift curve slope at the higher reduced frequencies; (5) peak drag coefficient for the higher reduced frequencies not at $\alpha=90^{\circ}$ but at some smaller value, say $\alpha=70^{\circ}$; and (6) departure from the static lift and drag values even at very small reduced frequencies, in this case $\mathrm{K}=0.01$. 

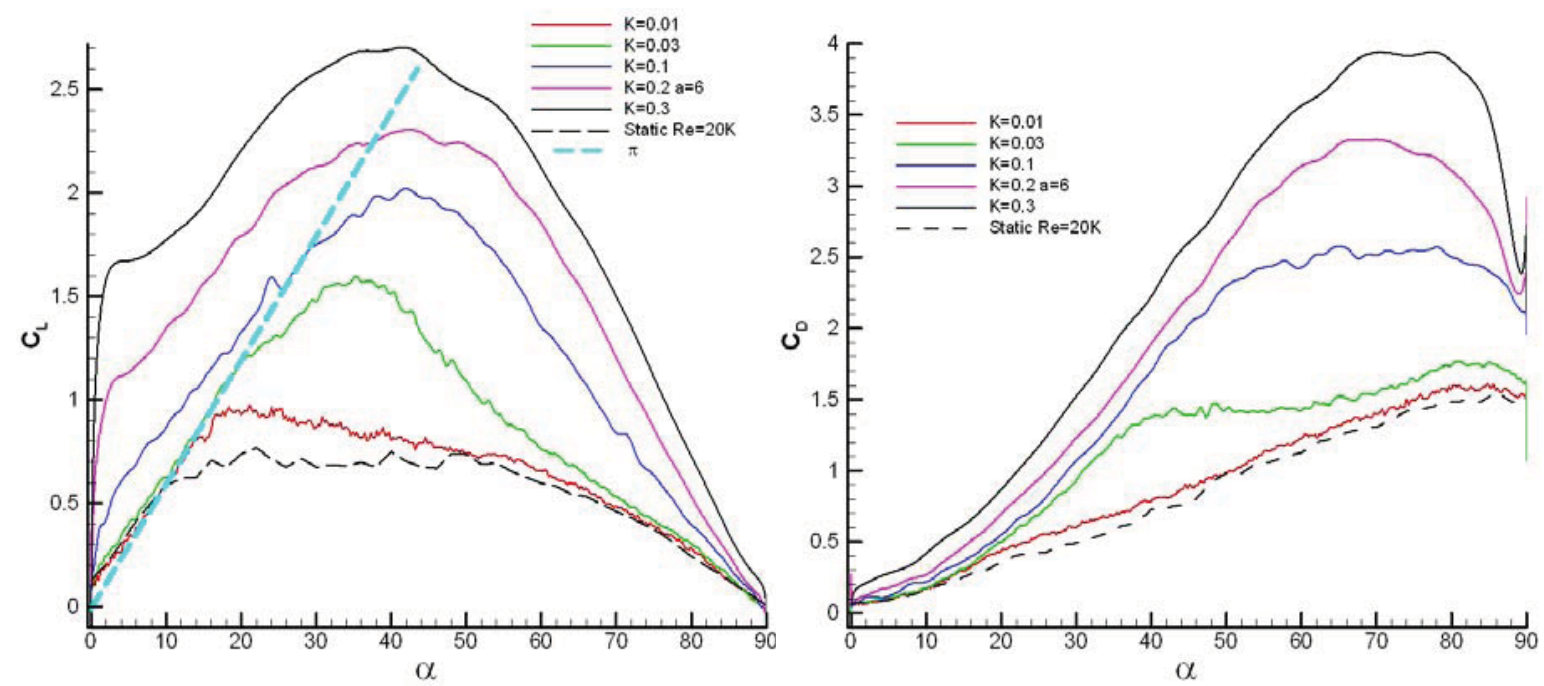

Figure 10. $A R=2$ rectangular plate lift coefficient (left) and drag coefficient (right), $K=0.01$ through $0.3, \operatorname{Re}=20 \mathrm{~K}, \alpha=0^{\circ}$ through $90^{\circ}$ linear pitch ramp, pivot at $\mathrm{x} / \mathrm{c}=0.25$.

In light of the differences in flowfield results between the rectangular $A R=2$ plate in Figure 8 and the Zimmerman $\mathrm{AR}=2$ plate in Figure 9, the similarities in lift and drag coefficient between the two in Figure 11 are perhaps surprising. One speculative possibility is that the rate-effects that dominate high-frequency motion attenuate dependency on planform shape, though evidently not on aspect ratio.
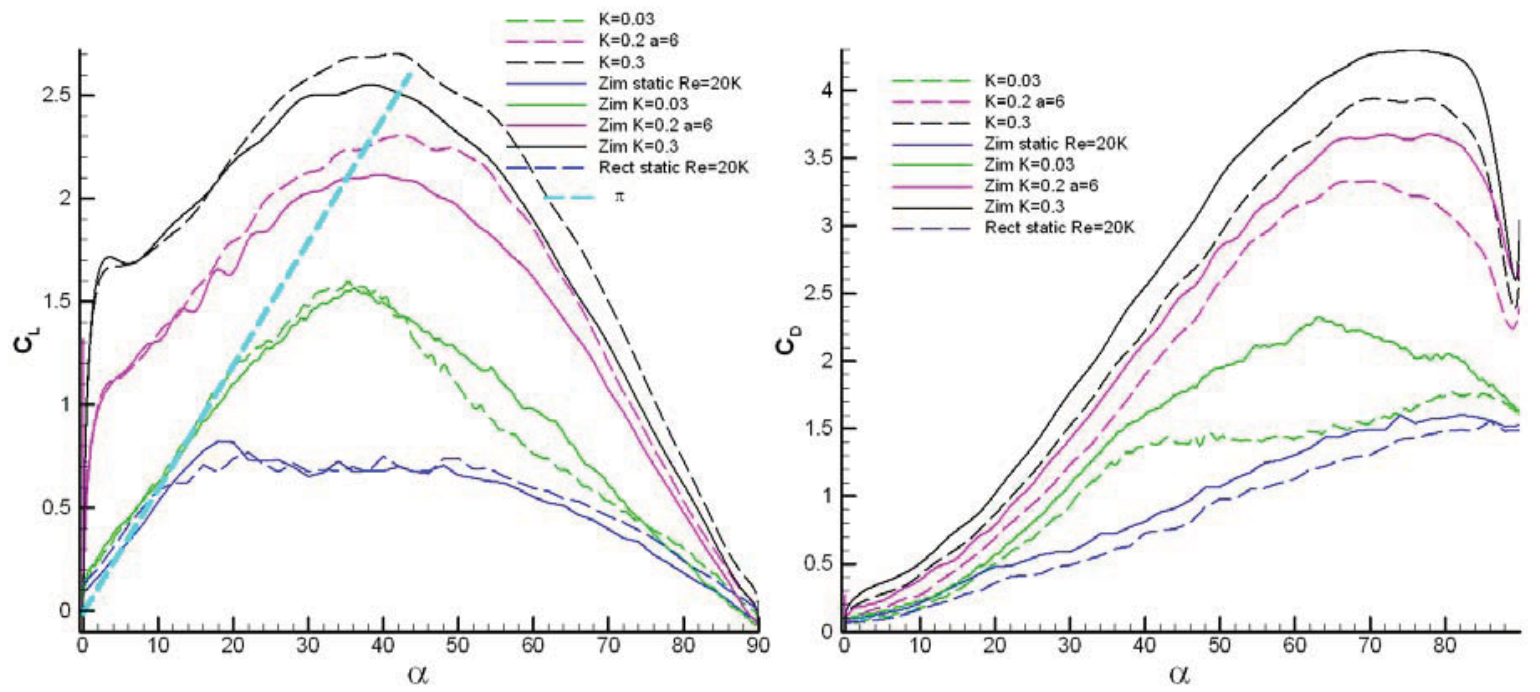

Figure 11. Comparison of $\mathrm{AR}=2$ rectangular plate (dashed lines) and Zimmerman plate (solid lines) lift coefficient (left) and drag coefficient (right), $K=0.03$ through $0.3, \operatorname{Re}=20 \mathrm{~K}, \alpha=0^{\circ}$ through $90^{\circ}$ linear pitch ramp, pivot at $\mathrm{x} / \mathrm{c}=\mathbf{0 . 2 5}$.

Aspect ratio dependency is shown in Figure 12, comparing the $2 \mathrm{D}$ and $\mathrm{AR}=2$ rectangular plate static lift and drag, and results at $K=0.03$ and $K=0.20$. Before the static stall break $\left(\alpha \leq 9^{\circ}\right)$, the lift curve slopes of the $A R=2$ plate and $2 \mathrm{D}$ plate follow the steady result; at $A R=2$, lifting-line theory and slender-wing theory both state that the lift curve slope should be half of the 2D case, and this is indeed the case in the measured lift coefficient. However, the post-stall behavior does not follow a simple theoretical relationship. In the dynamic cases, the $\mathrm{AR}=2$ plate generally stalls later and at a lower maximum lift coefficient than does the 2D plate. Stall is also smoother, in the sense of a broader peak near max lift. This is consistent with quasi-steady intuition for low aspect ratio 
wings. However, there is no obvious relation in going from $2 \mathrm{D}$ to $\mathrm{AR}=2$ in explaining peak lift coefficient decline. Turning to drag coefficient, in the static case, the AR=2 plate has a lower drag coefficient at post-stall angles of attack. This is consistent with the literature for flat plates ${ }^{22}$; the accepted value for time-averaged static drag coefficient for a $2 \mathrm{D}$ wall-normal flat plate is around 2.0, while for an $\mathrm{AR}=2$ wall normal plate the drag coefficient is near 1.5. That the present measurements comport with the accepted values is evidence - but not conclusive proof - that blockage in the test section is acceptably small. The trend of the $\mathrm{AR}=2$ plate having lower drag coefficient than the $2 \mathrm{D}$ plate continues for the dynamic cases, although for $\mathrm{K}=0.03$ the $\mathrm{AR}=2$ plate inexplicably has higher drag for $30^{\circ} \leq \alpha \leq 40^{\circ}$
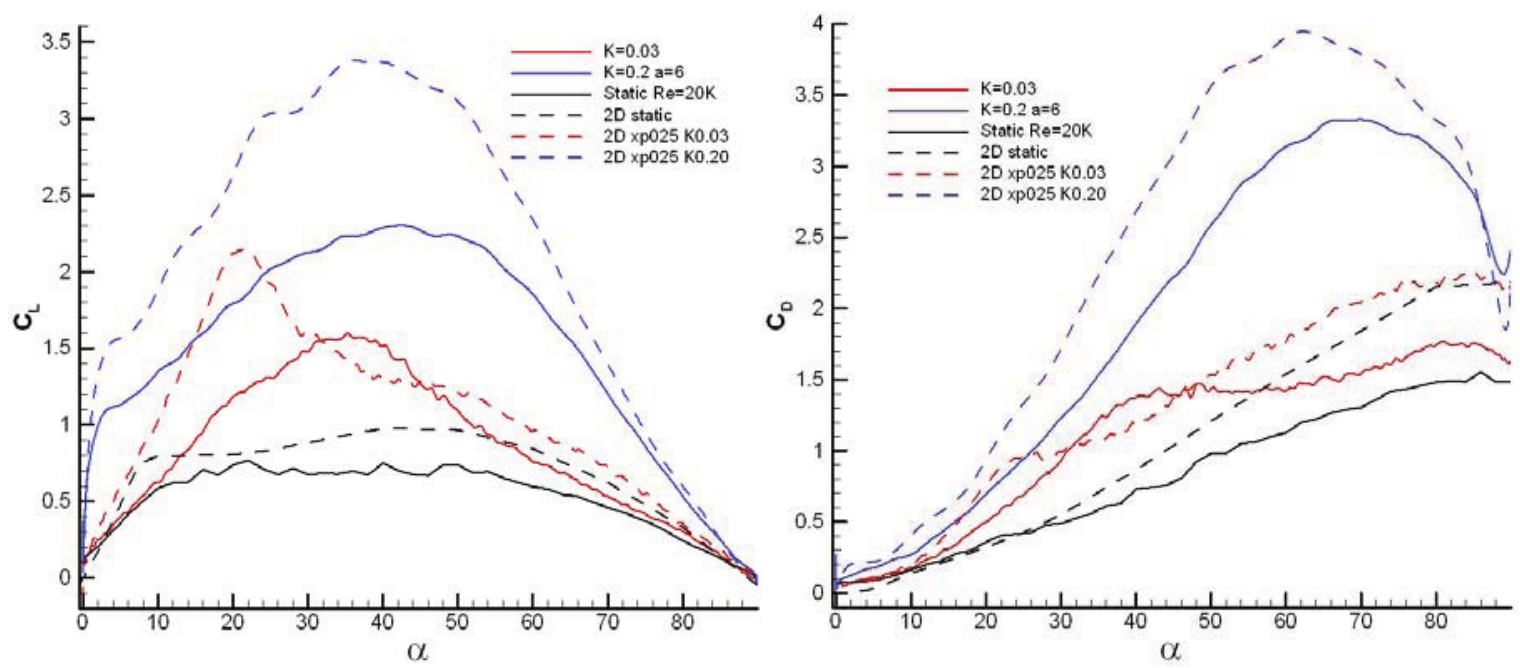

Figure 12. Comparison between $2 \mathrm{D}$ plate (dashed lines) and $\mathrm{AR}=2$ rectangular plate (solid lines), lift coefficient (left) and drag coefficient (right), pivot about $x / c=0.25, \operatorname{Re}=20 \mathrm{~K}$.

We next consider the role of acceleration and noncirculatory force, but considering different motion smoothing transients. Ol et al. ${ }^{23}$ compared a smoothed linear pitch ramp-holdreturn maneuver, $\alpha=0^{\circ}-45^{\circ}-0^{\circ}$, with a $45^{\circ}(1-\cos (\alpha))$ function, such that the two would have identical peak pitch rates. The idea was to compare noncirculatory contributions, as the cosine function is always accelerating, while the smoothed linear function is accelerating only during the smoothing. A similar approach can be taken for the $0^{\circ}-90^{\circ}$ pitch ramp, by varying the smoothing parameter, " $a$ "; this is shown for $\mathrm{K}=0.20$ in Figure 13. For $a=1$, the motion is nearly sinusoidal, and the startup/stopping transients have become subsumed by nearly sinusoidal acceleration throughout the motion. Therefore the noncirculatory spike in lift at startup, and in drag at motion cessation, are completely gone. The intermediate case between the original linear ramp motion and the near-sinusoid, $a=2.8$, tracks closely with the ramp cases except in the startup transient in lift. Finally, the case $a=10$ is an even more aggressive startup transient, and it shows the strongest noncirculatory startup spike in lift. We note, however, that the $\mathrm{a}=6$ and especially $\mathrm{a}=10$ noncirculatory "spikes" are attenuated by the low-pass filtering. Adjusting the filtering to higher wave number would unfortunately produce more apparent waviness in the lift and drag histories during the unaccelerated portion of the motion.

The relatively high overlap of all of the curves in Figure 13 is evidence of the success of linear superposition: circulatory and noncirculatory lift contributions are linearly additive. And as expected, the noncirculatory affects are present only when the model's acceleration is nonzero. 

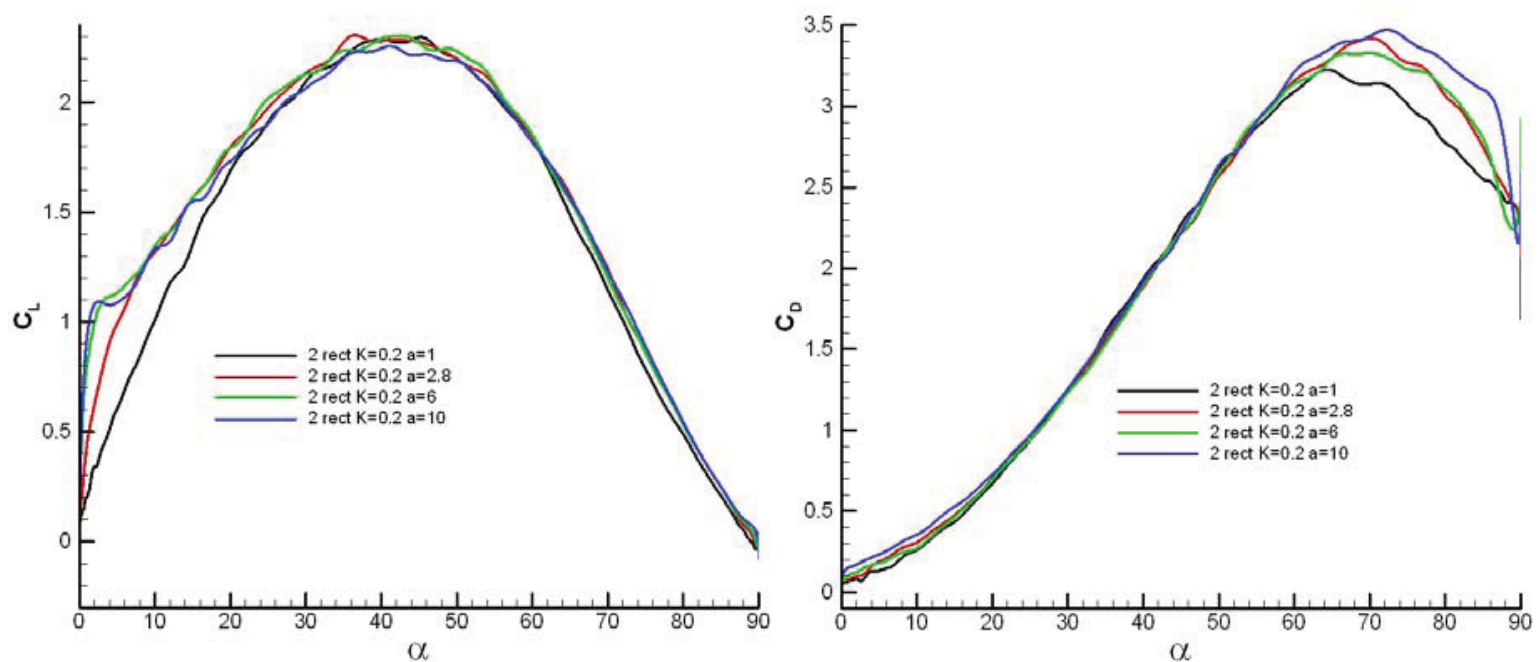

Figure 13. Survey of acceleration effects, $K=0.20, A R=2$ rectangular plate, pivot about $x / c=0.25$, $R e$ $=20 \mathrm{~K}$. " $\mathrm{a}=1$ " is a sinusoidal motion, while " $\mathrm{a}=6$ " repeats the smoothing in all of the above figures.

As points of departure from this work, it is worth mentioning that parameter studies of aspect ratio, reduced frequency, pivot point, planform shape, mounting arrangement and so forth are in principle unbounded and amorphous; one could choose any of a number of possibility to elucidate this or that trend. Two key points, however, emerge: first, what sort of lags does one find in the aerodynamic force coefficient response vs. the flowfield response? It does appear, for example from the progression of peak lift to higher and higher angle of attack with increasing reduced frequency, that the force coefficient lag correlates with the lag in LEV development. But to quantify this would require more sophisticated velocimetry method, tracking the evolution of LEV and TEV circulation. Second, to what extend can classical theoretical treatment of finite aspect ratio effects, so useful in static and quasi-steady problems, be extended to higher reduced frequency? This also is a question about lags, since presumably if the LEV, TEV and tip vortices developed at similar rates, finite aspect ratio effects would be the same at all reduced frequencies. As this is not the case, one is tempted to surmise that tip vortex growth or variation is slower than that of leading edge and trailing edge vortices. But substantiation of this too would require more sophisticated velocimetry.

\section{Conclusion}

Problems in low Reynolds number aerodynamics, with application to vehicle engineering, tend to be unsteady. From the facilities viewpoint, objectives include a fast and accurate rig for the relevant set of model motions, a force measurement capability that balances the competing objectives of good signal to noise ratio and high balance stiffness, and a flow visualization system that allows one to connect flowfield and force-measurement observations.

In the present work, we considered three flat plates in a water tunnel: nominally $2 \mathrm{D}$, rectangular with aspect ratio 2, and Zimmerman with aspect ratio 2. The imposed motion was a pure-pitch, at various pivot points and reduced frequencies. Broadly, high motion rate produces two important departures from the quasi-steady response: noncirculatory forces associated with fluid acceleration, and large positive increments in both lift and drag, likely associable with the rate of motion. Planform differences - between rectangle and Zimmerman - were seen to be small. Quasi-steady lifting line theory explains the trends in lift coefficient in going from 2D to low aspect ratio plates, but only for the slower rates of motion. Leading edge vortex growth and subsequent 
shedding, conceptually so dominant in high angle of attack problems, is seen to correlate with peaks in lift coefficient.

\section{References}

${ }^{1}$ Lentink, D., and Dickinson, M.H. "Rotational Accelerations Stabilize Leading Edge Vortices on Revolving Fly Wings". J. Exp. Biol. Vol. 212, pp. 2705-2719, 2009.

${ }^{2}$ Lua, K.B., Lai, K.C., Lim, T.T., and Yeo, K.S. "On the Aerodynamic Characteristics of Hovering Rigid and Flexible Hawkmoth-like Wings". Experiments in Fluids, Vol. 49, No. 6, pp. 1263-1291, 2010

${ }^{3}$ Singh, B., and Chopra, I. "Insect-Based Hover-Capable Flapping Wings for Micro Air Vehicles: Experiments and Analysis". AIAA Journal, Vol. 46, No. 9, pp. 2115-2135, 2008.

${ }^{4}$ Usherwood, J.R., and Ellington, C.P. "The Aerodynamics of Revolving Wings I. Model Hawkmoth Wings". J. Exp. Biol. Vol. 205, pp. 1547-1564, 2002

${ }^{5}$ Jones, A., and Babinsky, H. "Unsteady Lift Generation on Rotating Wings at Low Reynolds Numbers". Journal of Aircraft, Vol. 47, No. 3, pp. 1013-1021, 2010.

${ }^{6}$ Rival, D., Manejev, R., and Tropea, C. "Measurement of Parallel Blade-Vortex Interaction at Low Reynolds Numbers". Experiments in Fluids, Vol. 49, No. 1, pp. 89-99, 2010.

${ }^{7}$ Hart, A., and Ukeiley, L. "Low Reynolds Number Unsteady Aerodynamic over a Pitching-Plunging Flat Plate". AIAA 2010-0387.

${ }^{8}$ Brzozowski, D., Culp, J., Kutay, A., Muse, J., and Glezer, A. "Closed-Loop Aerodynamic Flow Control of a Free Airfoil". AIAA 2008-4323

${ }_{9}^{9}$ Bansmer, S., Radespiel, R., Unger, R., Haupt, M., and Horst, P. "Experimental and Numerical FluidStructure Analysis of Rigid and Flexible Flapping Airfoils". AIAA Journal, Vol. 48, No. 9, pp. 1959-1974, 2010.

${ }^{10}$ Baik, Y.S., Rausch, J., Bernal, L., and OL, M. "Experimental Investigation of Pitching and Plunging Airfoils at Reynolds Number between $1 \times 10^{\wedge} 4$ and 6x10^4". AIAA 2009-4030

${ }^{11}$ Visbal, M. "Numerical Simulation of Deep Dynamic Stall of a Plunging Airfoil". AIAA 2010-4458

${ }^{12}$ OL, M., Bernal, L., Kang, C.-K., and Shyy, W. "Shallow and Deep Dynamic Stall for Flapping Low Reynolds Number Airfoils". Experiments in Fluids, Vol. 46, Issue 5, pp. 883-901, May 2009.

${ }^{13}$ Eldredge, J.D., Toomey, J., and Medina, A. "On the Roles of Chord-Wise Flexibility in a Flapping Wing with Hovering Kinematics". Journal of Fluid Mechanics, Vol. 659, pp. 94-115, Sept. 2010.

${ }^{14}$ Koochesfahani, M.M., and Smiljanovski, V. "Initial Acceleration Effects on Flow Evolution around Airfoils Pitching to High Angle of Attack.” AIAA Journal, Vol. 31, No. 8, pp. 1529-1531, 1993.

${ }^{15}$ Reich, G., and Wojnar, O., and Albertani, R. "Aerodynamic Performance of a Notional Perching MAV Design". AIAA 2009-0063.

${ }^{16}$ Granlund, K., OL, M., Garmann, D., Visbal, M., and Bernal, L. "Experiments and Computations on Abstractions of Perching". AIAA 2010-4943.

${ }^{17}$ Laitone, E.V., "Wind Tunnel Tests of Wings at Reynolds Numbers Below 70,000," Experimental Fluids, Vol. 23, 1997, pp. 405-409.

${ }^{18}$ Mueller, T.J. "Low Reynolds Number Vehicles". AGARDograph No. 288, AGARD-AG-288.

${ }^{19}$ Buchmann, N., Buchner, A.-J., Kilany, K., Atkinson, C., and Soria, J. "Multi-Component - MultiDimensional PIV Measurements for a Flat-Plate Motion.

${ }^{20}$ Yilmaz, T., OL, M., and Rockwell, D. "Scaling of Flow Separation on a Pitching Low Aspect Ratio Plate". Journal of Fluids and Structures, Vol. 26, Issue 6, pp. 1034-1041, August 2010.

${ }^{21}$ Baik, Y.S., Aono, H., Rausch, J., Bernal, L., Shyy, W., and OL, M. "Experimental Study of Impulsively Rotated Flat Plate at Low Reynolds Number". AIAA 2010-4462.

${ }^{22}$ Hoerner, S.F. Fluid-Dynamic Drag. Hoerner Fluid Dynamics, PO Box 342, Brick Town, NJ, 08723, 1965.

${ }^{23}$ OL, M., Altman, A., Eldredge, J., Garmann, D., and Lian, Y. "Summary of Progress on Pitching Plates: Canonical Problems in Low-Re Unsteady Aerodynamics". AIAA-2010-1085 